Pozo-Vicente, Cristina; Aguaded-Gómez, José Ignacio (2012). El programa de movilidad ERASMUS: motor de la adquisición de competencias interculturales. Revista de Investigación Educativa, 30 (2), 441-458.

\title{
EL PROGRAMA DE MOVILIDAD ERASMUS: MOTOR DE LAADQUISICIÓN DE COMPETENCIAS INTERCULTURALES
}

\author{
Cristina Pozo-Vicente \\ Universität Bonn (Alemania) \\ José Ignacio Aguaded-Gómez \\ Universidad de Huelva (España)
}

\section{RESUMEN}

El programa de movilidad Erasmus, enmarcado en el contexto de la educación superior europea, no solo fomenta el aprendizaje de la lengua extranjera, sino que además promueve el conocimento físico de los estados miembros, el entendimiento y la cohesión entre miembros pertenecientes a diferentes culturas, lo que da conciencia de ciudadanía europea entre la población estudiantil. El objetivo de nuestro trabajo de investigación se centra en identificar en qué medida la estancia en el extranjero promueve la adquisición de competencia intercultural. El modelo de investigación adoptado es multimetódico y está basado en la aplicación de una metodología cuantitativa y cualitativa. Los instrumentos aplicados son el cuestionario-encuesta y el foro de discusión. El universo y la muestra de nuestro estudio es el circunscrito a los estudiantes Erasmus alemanes y españoles que durante el curso académico 2009/10 realizaron una estancia en universidades alemanas y españolas. El análisis de los resultados y las conclusiones aportan una información valiosa y de carácter descriptivo sobre la adquisición de competencias interculturales dentro del Espacio Europeo de Educación Superior.

Palabras clave: Programa de movilidad Erasmus; Educación superior; Estudiantes Erasmus alemanes y españoles; Competencias interculturales.

\footnotetext{
Correspondencia:

Cristina Pozo-Vicente, Universität Bonn (Alemania).
} 


\section{ERASMUS MOBILITY PROGRAMME: DRIVING FORCE OF THE ACQUISITION OF INTERCULTURAL COMPETENCIES}

\section{ABSTRACT}

The Erasmus student Mobility Programme plays an important role in the process of integration and consolidation of the European Union. It also promotes foreign language learning, learning about other Member States, and understanding of and unity among people from different cultures, which in turn increases awareness of European citizenship among students. Our main goal is to analyze in detail the acquisition of intercultural competencies of German and Spanish Erasmus students before and during their stay in a foreign country. We applied a multimethod model (or mixed method) based on both quantitative and qualitative approaches. The two instruments for data collection were a questionnaire and a focus group.

The sample group participating in our research were German and Spanish Erasmus students, who studied abroad during the academic year 2009/10 at German and Spanish universities. The analysis of results and the conclusions of our research provide important descriptive information about the state of affairs of foreign language learning and the use of the internet within the EHEA.

Keywords: Erasmus student Mobility Programme; Higher Education; German and Spanish Erasmus students; Intercultural competencies.

\section{FUNDAMENTACIÓNTEÓRICA}

\section{I.I. El programa de movilidad Erasmus}

El nombre del programa Erasmus es el acrónimo del nombre oficial en inglés «European Community Action Scheme for the Mobility of University Students» y se hizo coincidir con el nombre del filósofo y teólogo Erasmo de Rotterdam.

El programa Erasmus se creó en 1987 y, en la actualidad, forma parte del Programa de Aprendizaje Permanente. Su objetivo principal es el fomento de la construcción de un Espacio Europeo de Educación Superior.

\section{I.2. La competencia intercultural}

Los primeros estudios que definieron el concepto de «competencia intercultural» se basaron en descripciones de las características de las personas que vivían fuera de sus fronteras nacionales y de componentes que ayudaban a entender la competencia intercultural. En 1955, las investigaciones de Lygaards, sobre los estudiantes noruegos que pasaban una estancia en Estados Unidos, marcaron el comienzo de los análisis explorativos, seguida de la realizada por Stahl (1998) sobre enviados alemanes a Japón y EEUU (Bolten, 2007). Se establecieron de forma progresiva tipologías (p.ej. Ruben, 1976; Brislin, 1981) que definían la competencia intercultural como una suma de componentes. Entre sus características cabe resaltar: la «empatía», la «tolerancia» a la «ambigüedad», el «self-oriented role behaviour» (Ruben, 1976), la «conciencia cultu- 


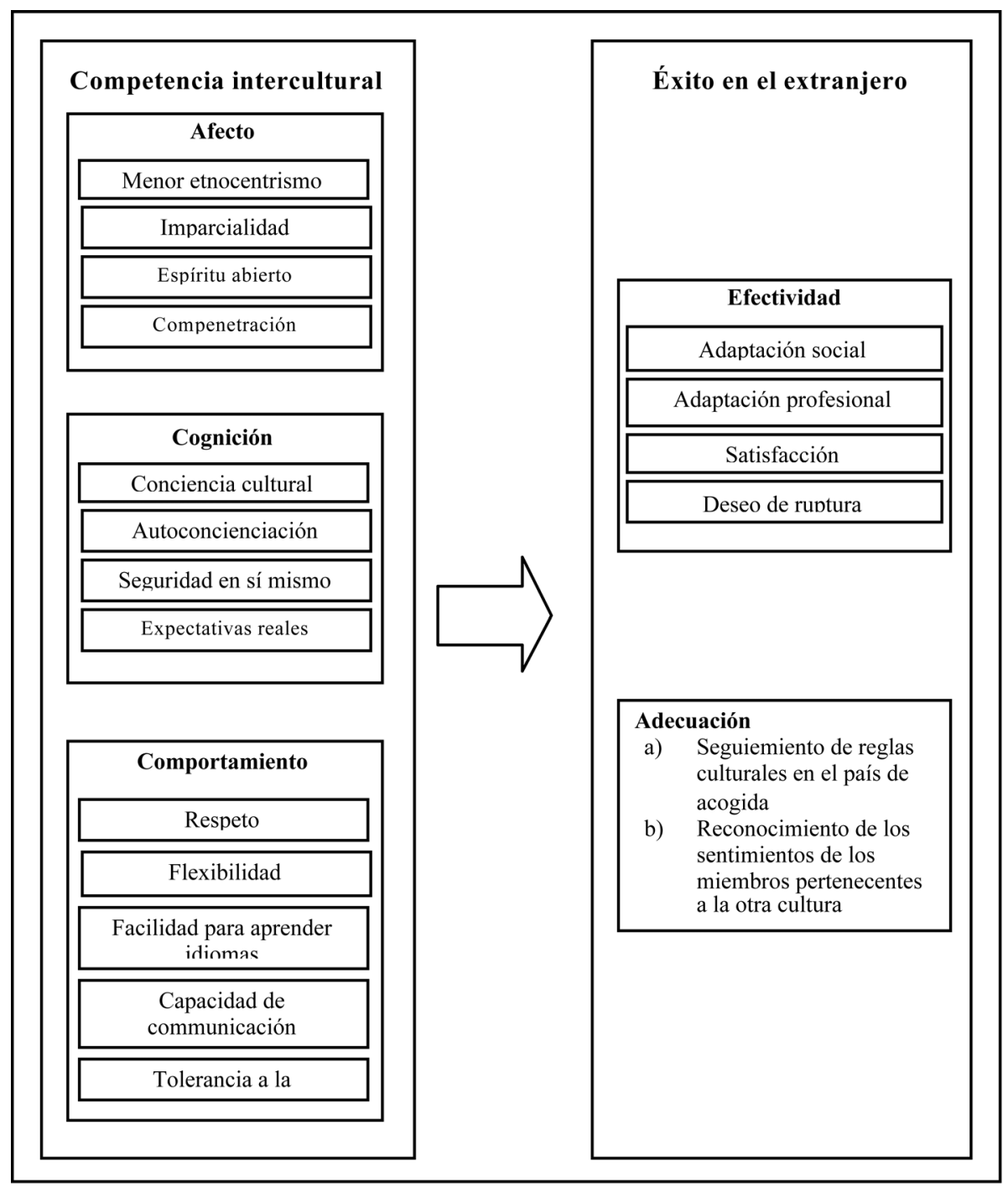

FIGURA 1.1

MODELO DE LA ESTRUCTURA DE LA COMPETENCIA INTERCULTURAL (MÜLLER Y GELBRICH, 2004: 794)

ral» (Triandis, 1977), el «espíritu abierto», el «respeto hacia las diferencias culturales» o la «competenetración en la interacción» (Chen, Starosta, 1997), y la «capacidad de adaptación» (Fritz, Möllenberg, Chen, 2004).

A partir de los años noventa una corriente de autores establecieron modelos estructurales o «Strukturmodelle» (Gertsen, 1990) que clasificaban la competencia intercultural en dimensiones afectivas, cognitivas y conativas y sistematizaban sus características. 
Müller y Gelbrich (2004) desarrollaron un modelo que complementaba la competencia intercultural con la «efectividad» y la «adecuación» como criterios externos (véase Figura 1.1).

Sin embargo, según Bolten (2007: 23) existe una relación de interdependencia entre las dimensiones cognitivas, afectivas y conativas y define la competencia intercultural como un producto sinérgico, resultado del permanente intercambio entre las dimensiones mencionadas, no como un concepto estructural, sino procesal.

$\mathrm{Su}$ modelo entiende la competencia intercultural como «una interacción global exitosa de acciones individuales, sociales, específicas y estratégicas en contextos interculturales» (Bolten, 2007: 24). Para este autor, un individuo es interculturalmente competente si es capaz de organizar de forma equilibrada la mencionada interacción sinérgica entre las dimensiones de acciones individuales, sociales, especializadas y estratégicas. Según Müller y Gelbrich (2004: 294) este equilibrio de las cuatro variables procesales representa un criterio externo de la competencia intercultural, que depende en gran parte del contexto. Mientras que el éxito de la actuación en una situación puede depender principalmente de la personalidad del individuo, en otros contextos culturales puede serlo, por ejemplo, la capacidad estratégica. Por lo tanto, la competencia intercultural contempla el conocimiento específico de la cultura.

En base a este enfoque de la competencia intercultural como proceso, Milton Bennett (1986) desarrolló un modelo denominado «Developmental Model of Intercultural Sensivity» (DMIS), basado en la psicología cognitiva, que describe cómo los individuos cambian sus relaciones y su percepción con la influencia de la/s cultura/s («cultural worldviews»); y que defiende un acercamiento a la cultura y la habilidad de cambiar las perspectivas culturales para desarrollar una «mente intercultural».

Según Milton Bennett (1986), existen dos fases: la etnocéntrica y la etnorrelativa. La fase etnocéntrica se divide, a su vez, en tres etapas: la negación, la defensa y la minimización. La etapa de negación implica el rechazo a las diferencias culturales. Para las personas que están en esa etapa (a veces puede durar toda la vida), el mundo es únicamente su propia experiencia. En la etapa de defensa, la persona se defiende frente a la diferencia cultural, tiende a percibir los estereotipos como una verdad y a polarizar cualquier discusión sobre las diferencias culturales; la propia cultura es la única y la verdadera, por lo que la otra cultura significa una amenaza a esa realidad. En la etapa de minimización, la persona minimiza las diferencias culturales, las cuales se subsumen en categorías familiares ya existentes y basadas en la cultura propia. En esta etapa, aún existe la posibilidad de que la persona quiera cambiar la actitud de los demás en función de sus expectativas.

La fase del etnorrelativismo se clasifica en tres etapas: aceptación, adaptación e integración. La etapa de aceptación no implica estar de acuerdo con las diferencias culturales, pero el juicio no es etnocéntrico en el sentido de negar la igualdad de la humanidad. Las personas aprenden a respetar las diferencias conductuales y de valores característicos a esa cultura subjetiva como el lenguaje, la conducta no verbal y los estilos de comunicación. En la etapa de adaptación, se amplía la visión propia y se consigue la empatía intercultural. Ese proceso requiere el conocimiento de la cultura propia y de sus diferencias con otras culturas. En la etapa de integración los sujetos amplían su capacidad de percibir los hechos en el contexto cultural para incluir la 
propia definición de identidad. Se trata de un proceso de cambio de perspectiva e implica un cambio de identidad.

Deardorff (2006), como Milton Bennett, defiende que la competencia intercultural forma parte de un proceso continuo de reflexión y que está relacionado con la situación, con una determinada relación intercultural y con el intercambio comunicativo entre personas de diferentes culturas. La define en base a la adquisición de determinados comportamientos y actitudes en contextos interculturales como el respeto, la curiosidad, la tolerancia, así como de las capacidades de acción y de reflexión crítica tales como analizar, interpertar, evaluar y observar. Además, afirma que el desarrollo de la competencia intercultural no se produce de forma natural, sino que tiene que ser un acto intencionado. Por eso, postula que es necesario el desarrollo de programas específicos en la educación formal orientados a la adquisición de esta competencia.

Löschmann (2001: 2) también defiende el proceso de reflexión para alcanzar la competencia intercultural y afirma que el factor clave es el trato adecuado de los estereotipos y de los prejuicios. Asegura que los procesos de percepción y de opinión están determinados por contenidos mentales esquematizados y considera que el prejuicio es más una subcategoría de una opinión (actitud) y que tiene menos carga emocional que el estereotipo. La Real Academia de la Lengua Española define prejuicio como «una opinión previa y tenaz por lo general desfavorable acerca de algo que se conoce mal», y estereotipo como «una imagen o idea aceptada comúnmente por un grupo o sociedad con carácter inmutable». En base a estas definiciones podemos decir que los estereotipos contienen una carga negativa o menos positiva. Por ejemplo, la disciplina se considera un aspecto positivo dentro del propio grupo, mientras que el grupo externo se considera una «actuación sin piedad».

Una solución a los efectos negativos del estereotipo es la capacidad de elaborar lo que Mead (1994) denomina la «generalización creativa» y Adler (1987) el «estereotipo efectivo». Adler (1987) sugiere que debemos utilizar el estereotipo como una forma inicial de acercarnos a la persona antes de tener información directa, lo que se entiende como «una primera hipótesis» sobre la persona para después ir modificándola en la relación interpersonal y en base al diálogo.

Mead (1994) define una serie de actitudes que caracterizan a la persona que sabe emplear de forma efectiva este estereotipo, al cual denomina de «generalización creativa». Se trata de un estereotipo dinámico por su capacidad de cambiar y es como una primera hipótesis, susceptible al cambio en la interacción con la otra cultura. Según Santacreu y Albert (2004), se trata de un proceso de aculturación dinámico y positivo que fomenta la interculturalidad, los valores positivos del respeto, la aceptación y el aprendizaje mutuo.

Por el contrario, Mead (1994) denomina «estereotipos estáticos» a los que resultan de las actitudes de la persona que no utiliza el estereotipo de generalización creativa de forma efectiva. Generalmente, se recibe de otra persona o grupo, no es resultado de su propia creación y se selecciona la información que concuerda con el estereotipo reforzándolo con información incompleta y desviada.

Por lo tanto, categorizar los estereotipos nos ayuda a entender lo desconocido, lo incomprensible y lo extranjero. Si la activación de estereotipos depende de la volun- 
tad del aprendiz, su motivación y su disposición a adoptar una actitud abierta hacia nuevos modelos mentales desempeñan un papel importante (Santacreu y Albert, 2004).

\subsection{La estancia Erasmus y la competencia intercultural}

La mayoría de estudios como el de Deardorff (2004) y el de Straffon (2003) coincide en señalar que las experiencias interculturales permiten a los estudiantes conocer los comportamientos y progresar en las habilidades de la competencia comunicativa intercultural y afirma que existe una relación proporcional positiva y directa entre el tiempo de estancia en el extranjero y el grado de sensibilidad intercultural adquirido.

Esta sensibilidad intercultural se intensifica en contextos reales como, por ejemplo, el que es objeto de estudio en este trabajo de investigación. En la estancia en el extranjero, el estudiante tiene que hacer frente a un proceso continuo de descodificación y de negociación de la otra cultura, lo que conlleva establecer comparaciones entre lo cercano y lo distante, lo similar y lo distinto. Ese tipo de ejercicio permanente permite desarrollar estrategias de adaptación al nuevo contexto y llevar a cabo un proceso de cambio y de ajuste sociocultural.

Según Santacreu y Albert (2004), la estancia en el extranjero ha logrado su objetivo cuando al final de un proceso mental y de aprendizaje se pueden reconocer los estereotipos sociales y culturales como etapas hacia el camino de una perspectiva más compleja que su temporalidad e insuficiencia. Cuando nos hemos apropiado del «Otro» y ese «Otro» pasa a formar parte de nuestra propia identidad, ya no existe lugar para los estereotipos culturales.

\section{METODOLOGÍA}

Nuestro tema de investigación parte del estudio previo realizado durante el curso 2007/08 y que tenía como muestra de estudio los estudiantes de Hispánicas de la Universidad de Bonn que habían permanecido en España gracias a una beca Erasmus. En ese estudio se aplicó básicamente el cuestionario como técnica útil para evaluar las percepciones, expectativas y problemas de los participantes durante su estancia en el país extranjero.

La estructura general de la investigación la planteamos partiendo de la relación existente entre las variables de la investigación y mediante la aplicación de dos herramientas para la recogida de datos que posibilitaran abordar el fenómeno definido.

\section{I. Objetivos}

El objetivo general es conocer la adquisición de competencias interculturales de los estudiantes Erasmus alemanes y españoles antes y durante su estancia en el extranjero. Como objetivos específicos se pretende identificar en qué medida la estancia Erasmus sirve para promover actitudes como la empatía, el respeto y la aceptación hacia las otras culturas; y analizar cómo la estancia Erasmus a superar estereotipos y prejuicios sobre la otra cultura. 


\subsection{Proceso investigador}

En nuestro proceso de investigación hemos tenido en cuenta las fases preparatoria, de investigación y final propuestas por Gunzenhäuser y Haas (2006). La fase preparatoria se ha centrado en el período inicial del trabajo, en la ampliación del tema y en la temporalización, así como en el diseño de su fundamentación teórica. La fase de investigación se ha basado principalmente en el trabajo de campo y en el análisis de los datos. Tras el diseño y la estructuración de la investigación, hemos accedido al campo donde hemos aplicado los instrumentos elaborados para la recogida de datos, la «encuesta-cuestionario» y el «foro». En la aplicación de las herramientas de recogida de datos diferenciamos dos sub-fases. La sub-fase A (de octubre de 2009 a febrero de 2010) se ha caracterizado por la publicación del cuestionario (en dos lenguas: español y alemán) en internet y la participación de los estudiantes de las universidades invitadas. La sub-fase B (de marzo de 2010 a septiembre de 2010) se ha centrado en la apertura del foro de discusión en internet y la participación de los estudiantes seleccionados para discutir sobre aspectos planteados en la encuesta-cuestionario.

El proceso de tratamiento de la información ha durado cuatro meses aproximadamente (de octubre de 2010 a enero de 2011) y las técnicas aplicadas han sido la cuantitativa y la cualitativa. Para el tratamiento de la información obtenida a través del cuestionario, se han codificado las variables de estudio, se han cuantificado los datos empleando el programa estadístico SPSS y Microsoft Excel para la elaboración de los gráficos; y se han interpretado los resultados obtenidos del tratamiento estadístico. En el caso de la información obtenida a través del foro de discusión, las intervenciones se han clasificado en base a los objetivos a los que respondían y, posteriormente, se han interpretado los datos mediante la reflexión hermenéutica. Finalmente, se ha realizado una aproximación de las técnicas de investigación y se han comparado los resultados.

La fase final ha implicado la redacción de las conclusiones a partir de la reflexión del análisis de los resultados, la elaboración de las limitaciones del estudio y las propuestas de mejora y la optimización y revisión del trabajo en su conjunto.

\subsection{Descripción de la muestra}

Se ha llevado a cabo un proceso de selección o muestreo estratégico voluntario, también denominado por Goetz y LeCompte (1988: 102) de «casos típico-ideal», y que se emplea en diseños de estudio de caso único en los que la recogida de información procede básica y casi exclusivamente de un informante-clave (estudiantes «Erasmus»).

Para delimitar la muestra, dado que esa población es numerosa y que los factores a considerar se caracterizan por su infinitud (país de origen, país de recepción, lenguas, estudios, etc.), hemos tomado como población de la muestra: a) estudiantes españoles que han realizado una estancia Erasmus en Alemania y, en concreto, de las universidades: Rheinische Friedrich-Wilhelms-Universität Bonn, Universität zu Köln, Freie Universität Berlin, RWTH Aachen University; y b) estudiantes alemanes con estancia Erasmus en España en las siguientes universidades: Universidad de Huelva, Universidad de Sevilla, Universitat Pompeu Fabra, Universitat de Barcelona y Universitat Autònoma de Barcelona. 
Para participar en la encuesta-cuestionario el requisito ha sido que los estudiantes cursaran sus estudios durante un semestre o un curso académico en alguna de las nueve universidades españolas o alemanas citadas. La muestra de nuestra investigación está formada por 188 estudiantes Erasmus.

En el caso del foro de discusión, se ha seleccionado previamente a los participantes de entre la población que ha colaborado en la encuesta-cuestionario. Los criterios de selección se han basado en la elección de 4 estudiantes Erasmus españoles y 4 estudiantes Erasmus alemanes que, en la cumplimentación de la encuesta-cuestionario, han mostrado más predisposición por colaborar en el estudio para, de ese modo, asegurar su continua participación en el foro de discusión.

\subsection{Instrumentos de recogida de datos}

Como ya se ha mencionado anteriormente, en nuestra investigación hemos recogido la información a partir de las respuestas de los informantes realizadas en las encuestascuestionarios y de la información obtenida en el foro de discusión.

\subsection{Fiabilidad y validez del instrumento}

El procedimiento metodológico se ha basado en la aplicación de una prueba piloto, validada por expertos, grupo formado por profesores de lenguas extranjeras y por estudiantes Erasmus que no ha formado parte de la muestra. Este grupo ha analizado el contenido de la encuesta-cuestionario y la correspondencia entre los indicadores y los ítems. Así mismo, han efectuado las correcciones y mejoras oportunas en relación a la redacción de los ítems. Éste ha sido el paso previo para el diseño definitivo de la encuesta-cuestionario que posteriormente se ha sometido a la prueba de confidencialidad.

Para encontrar dicho coeficiente: hemos aplicado la prueba piloto en 10 sujetos no pertenecientes a la muestra del estudio, pero con características equivalentes a la misma. Hemos efectuado el cálculo del coeficiente Alfa Cronbach y la interpretación de los valores, teniendo en cuenta la escala que sugiere Ruiz (1998). El resultado final de todo el proceso ha sido: la obtención de un coeficiente de confiabilidad del 0,93 que, según la escala de Ruiz, es de magnitud muy alta, lo que nos ha confirmado la fiabilidad de la encuesta-cuestionario.

\subsubsection{Proceso de recogida de datos}

El proceso de recogida de datos y sistematización de la información se ha caracterizado por ser el eje de nuestro trabajo. En primer lugar, hemos contactado con los coordinadores Erasmus de las universidades participantes: Universidad de Huelva, Universidad de Sevilla, Universitat Pompeu Fabra, Universitat de Barcelona, Universitat Autònoma de Barcelona, Rheinische Friedrich-Wilhelms-Universität Bonn, Universität zu Köln Freie Universität Berlin y RWTH Aachen Universität. Los propios coordinadores de cada centro han sido los encargados de enviar a los estudiantes Erasmus nuestra invitación a participar en el cuestionario. 
En cuanto a los cuestionarios, la recepción de las respuestas se ha generado automáticamente un correo electrónico de aviso de envío dirigido al buzón de los investigadores y un correo respuesta de la investigadora en agradecimiento a la colaboración de los participantes en el estudio. En el correo que ha recibido la investigadora se ha adjuntado el cuestionario completo en formato Excel para ser procesado con el paquete estadístico SPSS.

\subsubsection{Descripción de los instrumentos}

El cuestionario se ha orientado hacia un enfoque psicopedagógico y se ha configurado como una autoevaluación del propio aprendizaje. La encuesta-cuestionario presenta un conjunto de preguntas que se han considerado relevantes para el rasgo, característica o variable que son objeto de estudio y que están orientados a la medición y al diagnóstico (véase figura 2.1).

\begin{tabular}{|c|c|}
\hline Objetivos & Preguntas \\
\hline \multirow{7}{*}{$\begin{array}{l}\text { Identificar en qué medida la } \\
\text { estancia Erasmus ha servi- } \\
\text { do para promover actitudes } \\
\text { como la empatía, el respeto y } \\
\text { la aceptación hacia las otras } \\
\text { culturas }\end{array}$} & $\begin{array}{l}\text { ¿Crees que conocer la cultura alemana/española ha } \\
\text { influido/influye en la forma de ver tu propia cultura? }\end{array}$ \\
\hline & $\begin{array}{l}\text { ¿Crees que la cultura española/alemana influye/ ha } \\
\text { influido en tu forma de acercarte a la cultura alema- } \\
\text { na/española? }\end{array}$ \\
\hline & $\begin{array}{l}\text { ¿Crees que existen diferencias entre la cultura alema- } \\
\text { na y la española? }\end{array}$ \\
\hline & $\begin{array}{l}\text { En encuentros con alemanes/españoles, ¿adoptas } \\
\text { actitudes que prejuzguen los comportamientos, opi- } \\
\text { niones y hábitos culturales de los alemanes/ españo- } \\
\text { les? }\end{array}$ \\
\hline & $\begin{array}{l}\text { ¿Puedes entender actitudes y opiniones de la cultura } \\
\text { alemana/española distintas a las tuyas? }\end{array}$ \\
\hline & $\begin{array}{l}\text { ¿Crees que puedes ponerte en el lugar de los alema- } \\
\text { nes/españoles, es decir, escucharles y entender sus } \\
\text { problemas y motivaciones (empatía)? }\end{array}$ \\
\hline & $\begin{array}{l}\text { ¿Eres capaz de integrar en tu estilo de vida hábitos } \\
\text { de la cultura alemana/española (alimentación, forma } \\
\text { de vivir, pensar, actuar etc.)? }\end{array}$ \\
\hline $\begin{array}{l}\text { Analizar cómo contribuye la } \\
\text { estancia Erasmus a superar } \\
\text { estereotipos y prejuicios so- } \\
\text { bre la otra cultura }\end{array}$ & $\begin{array}{l}\text { ¿Crees que los estereotipos y prejuicios sobre la cul- } \\
\text { tura alemana/española son verdad? }\end{array}$ \\
\hline
\end{tabular}

FIGURA 2.1

PREGUNTAS DEL CUESTIONARIO RELACIONADAS CON LOS OBJETIVOS DEL ESTUDIO

RIE, vol. 30-2 (2012) 
El foro de discusión ha sido el instrumento de recogida de datos que ha complementado la técnica cuantitativa. Las opiniones de los ocho estudiantes participantes, sobre temas planteados en la encuesta-cuestionario, nos han aportado datos y matices que han posibilitado complementar la información necesaria para nuestro estudio. La web que hemos empleado para llevar a cabo el foro de discusión fue "Wikispace», aplicación que puede ser editada por múltiples lectores. Se han generado dos «wikis»: un foro de discusión para los estudiantes Erasmus españoles y otro para los alemanes: «Experiencia Erasmus» (http://experienciaerasmus.wikispaces.com) y «Erasmus Erfahrung» (http://erasmuserfahrung.wikispaces.com).

\section{ANÁLISISY DISCUSIÓN DE DATOS}

En primer lugar se presentan los resultados obtenidos del tratamiento estadístico de los cuestionarios sobre las competencias interculturales $\mathrm{y}$, posteriormente, se expone un resumen de las opiniones que los estudiantes aportaron en el foro de discusión.

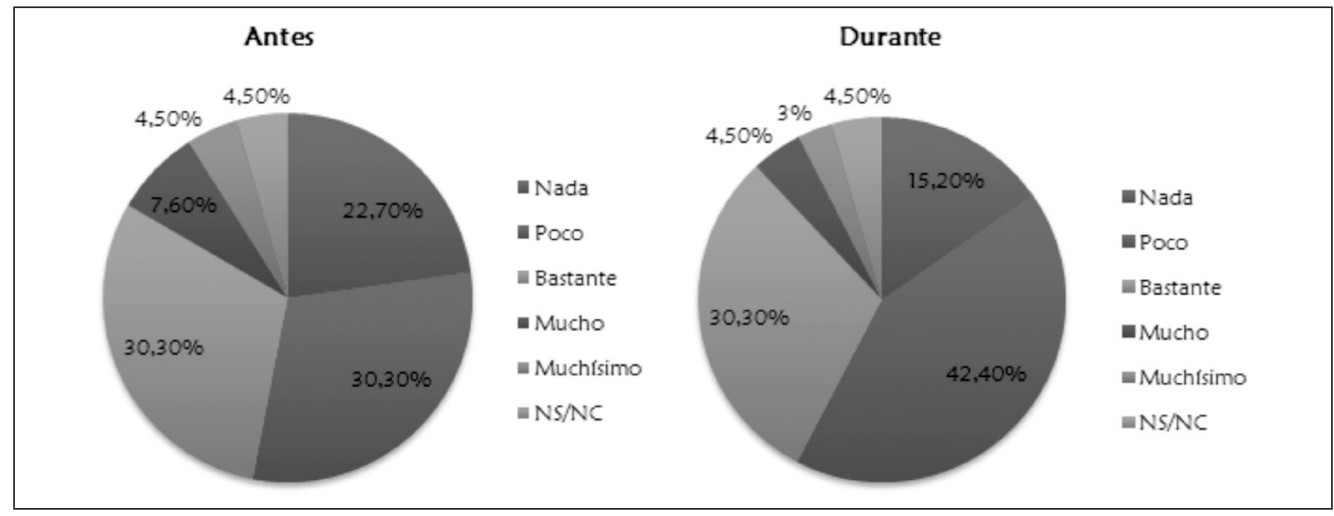

FIGURA 3.1

PORCENTAJE DE LAS RESPUESTAS DE LOS ESTUDIANTES ERASMUS ESPAÑOLES SOBRE LA VERACIDAD DE LOS ESTEREOTIPOS Y PREJUICIOS HACIA LA CULTURA ALEMANA

En la figura 3.1 se muestran los porcentajes de las respuestas de los estudiantes Erasmus españoles a la pregunta: «¿Crees que los estereotipos y prejuicios sobre la cultura alemana son verdad?». Los resultados muestran un aumento considerable, durante la estancia Erasmus y respecto al periodo anterior, de los porcentajes de «poco» y una disminución de los de «mucho»y «muchísimo».

La figura 3.2 muestra los porcentajes de las respuestas de los estudiantes Erasmus alemanes respecto a la pregunta: «¿Crees que los estereotipos y los prejuicios sobre la cultura española son verdad?». En el caso de los estudiantes alemanes, durante la estancia Erasmus disminuye, sobre todo, el porcentaje de «nada» y aumenta el porcentaje de «muchísimo».

En la figura 3.3 se observan los porcentajes de las respuestas de los estudiantes Erasmus españoles antes y durante el Erasmus respecto a la pregunta: « ¿En encuentros con alemanes adoptas actitudes que prejuzguen los comportamientos, opiniones y hábitos 


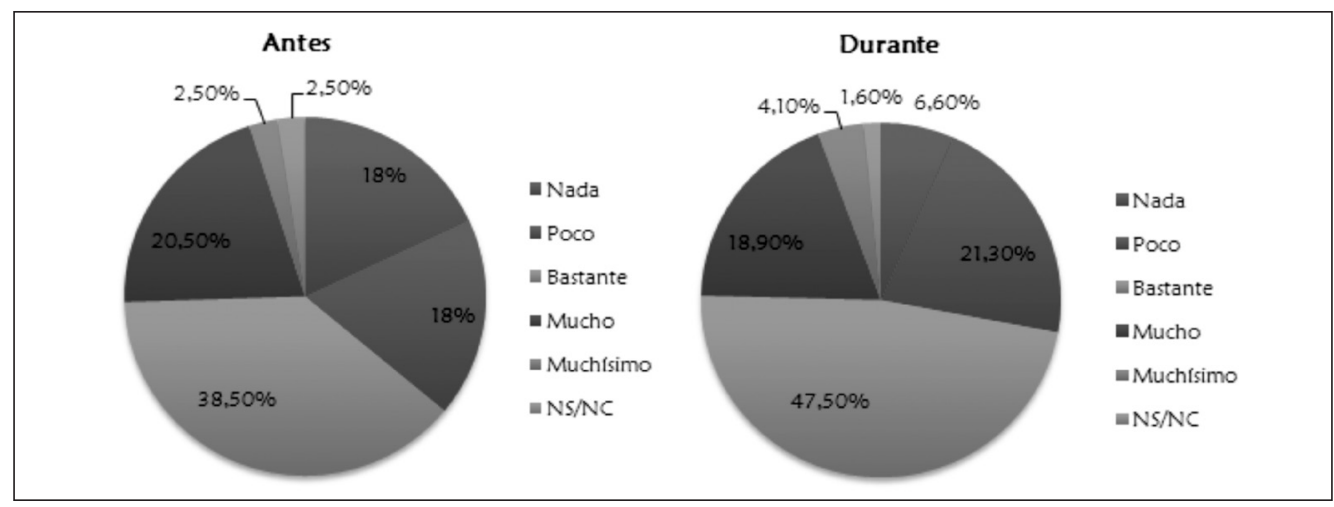

FIGURA 3.2

PORCENTAJE DE LAS RESPUESTAS DE LOS ESTUDIANTES ERASMUS ALEMANES SOBRE LA VERACIDAD DE LOS ESTEREOTIPOS Y PREJUICIOS HACIA LA CULTURA ESPAÑOLA

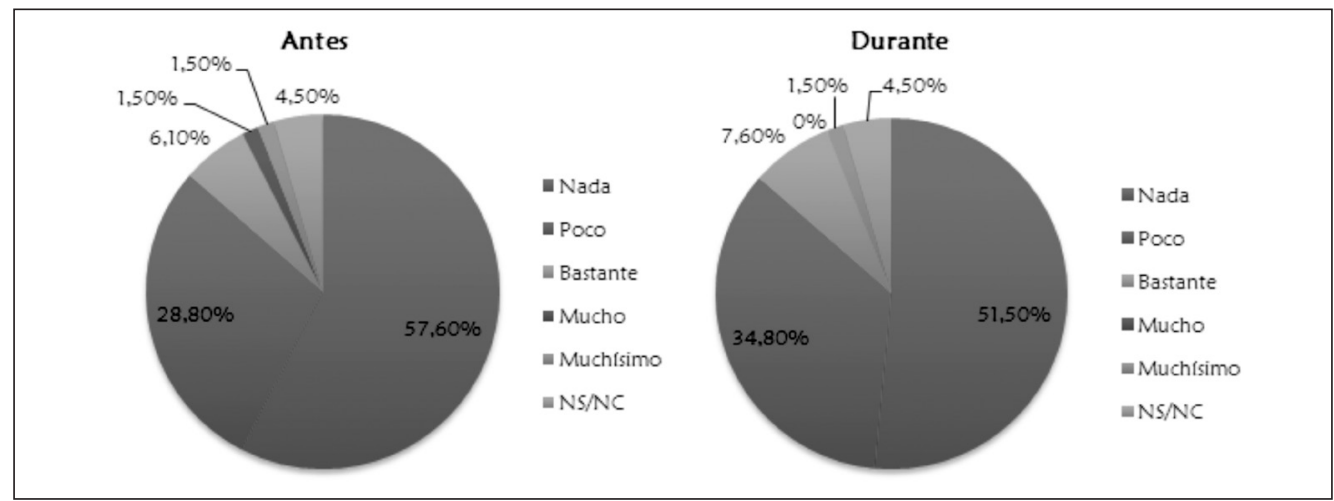

FIGURA 3.3

PORCENTAJE DE LAS RESPUESTAS DE LOS ESTUDIANTES ESPAÑOLES SOBRE LA ADOPCIÓN DE ACTITUDES QUE PREJUZGAN HÁBITOS CULTURALES DE LOS ALEMANES

culturales de los alemanes?». Cabe destacar que en ambos periodos la respuesta que tiene un mayor porcentaje y de forma clara es «nada», aunque en el segundo periodo disminuye ligeramente.

En la figura 3.4 se presentan los porcentajes de las respuestas de los estudiantes Erasmus españoles antes y durante la estancia Erasmus referentes a la pregunta: «¿En encuentros con españoles adoptas actitudes que prejuzguen los comportamientos, opiniones y hábitos culturales de los españoles?». Destacar que durante la estancia en el extranjero, los porcentajes de «poco»y «bastante» aumentan y el de «nada» disminuye, al igual que el de «mucho».

En la figura 3.5 se pueden observar los porcentajes de las respuestas de los estudiantes Erasmus españoles referente a la pregunta: «¿Puedes entender actitudes y opiniones de la cultura alemana distintas a las tuyas?». Durante la estancia Erasmus la respuesta de 


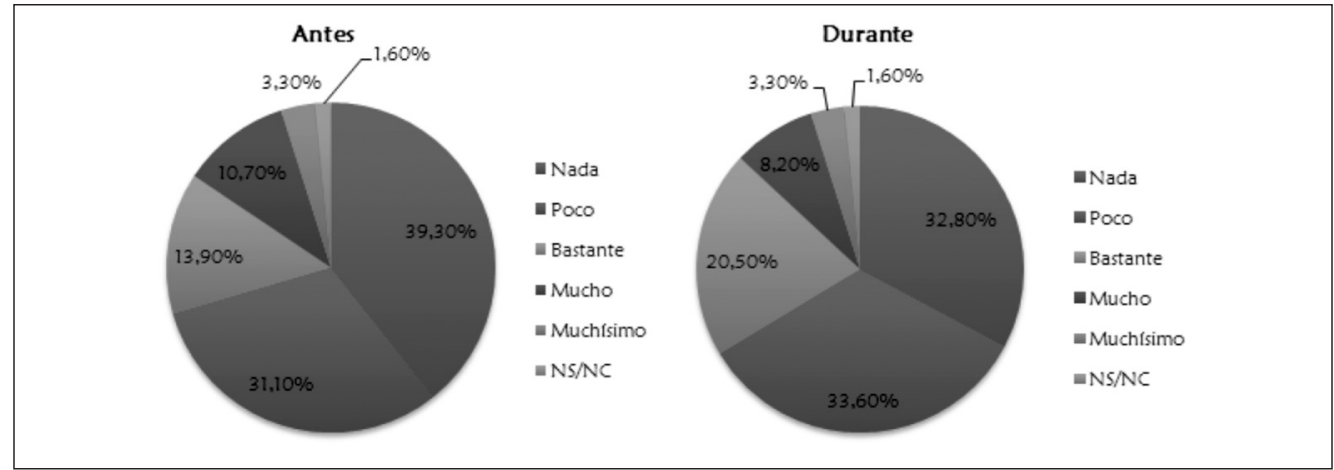

FIGURA 3.4

PORCENTAJE DE LAS RESPUESTAS DE LOS ESTUDIANTES ALEMANES SOBRE LA ADOPCIÓN DE ACTITUDES QUE PREJUZGAN HÁBITOS CULTURALES DE LOS ESPAÑOLES

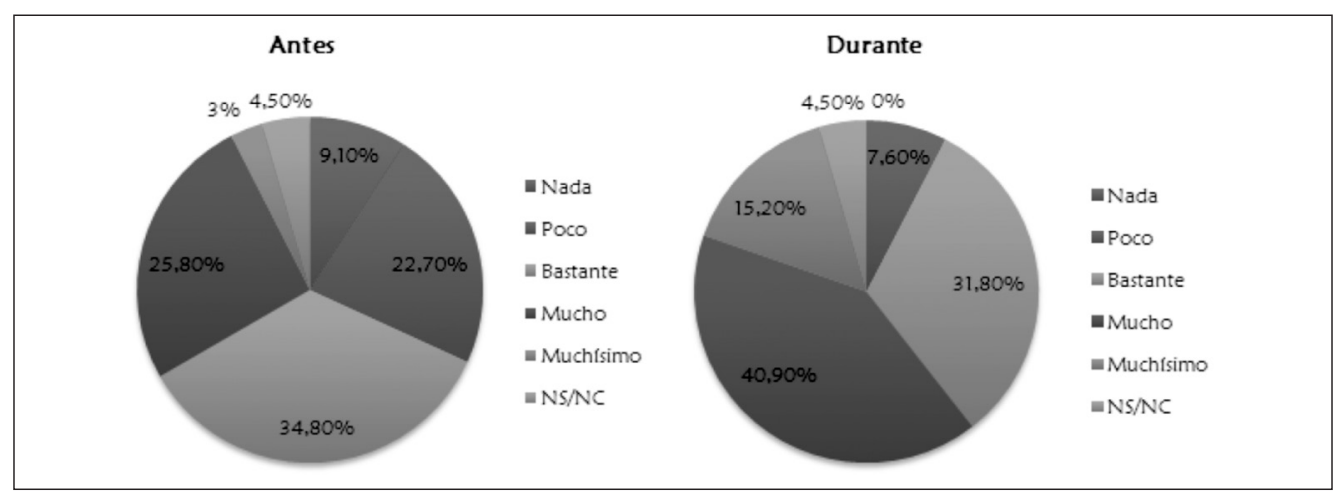

FIGURA 3.5

PORCENTAJE DE LAS RESPUESTAS DE LOS ESTUDIANTES ESPAÑOLES SOBRE LA COMPRENSIÓN DE ACTITUDES Y OPINIONES DE LA CULTURA ALEMANA DISTINTAS A LAS PROPIAS

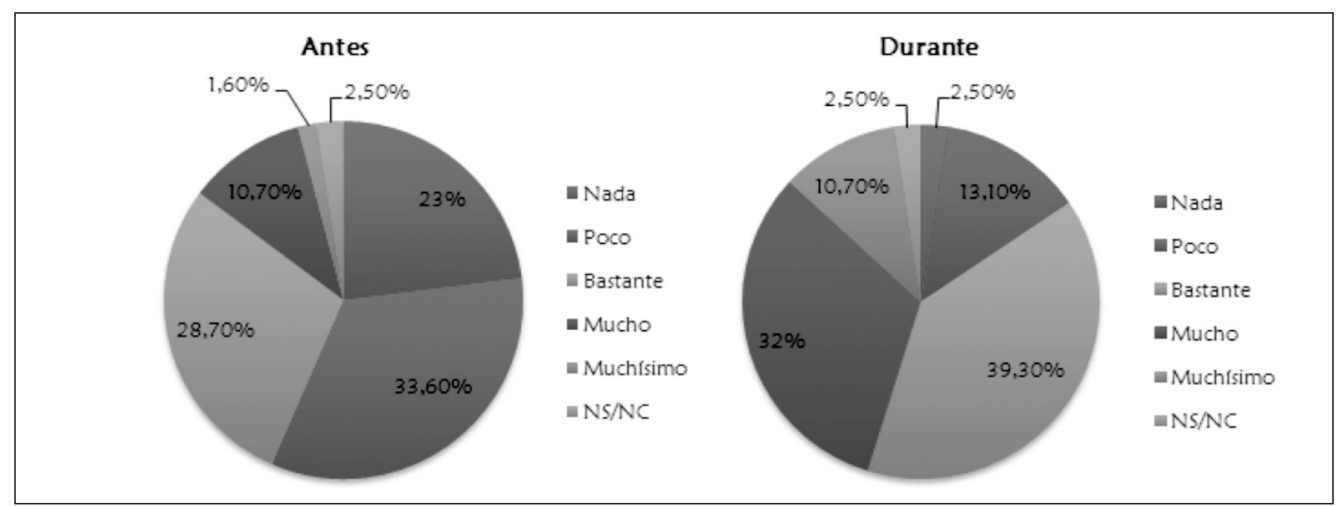

FIGURA 3.6

PORCENTAJE DE LAS RESPUESTAS DE LOS ESTUDIANTES ALEMANES SOBRE LA COMPRENSIÓN DE ACTITUDES Y OPINIONES DE LA CULTURA ESPAÑOLA DISTINTAS A LAS PROPIAS 
«mucho» aumenta, respecto al periodo anterior, de la misma forma que la de «muchísimo», mientras que las de «bastante», «poco» $\mathrm{y}$ «nada» disminuyen considerablemente.

En la figura 3.6 se presentan los porcentajes de las respuestas de los estudiantes Erasmus correspondientes a ambos periodos referidos a la pregunta: « ¿Puedes entender actitudes y opiniones de la cultura española distintas a las tuyas?». En la estancia en el extranjero se produce un cambio rotundo en el que «poco» $\mathrm{y}$ «nada» disminuyen considerablemente, y en el que «bastante», «mucho» $\mathrm{y}$ «muchísimo» aumentan de forma notable.

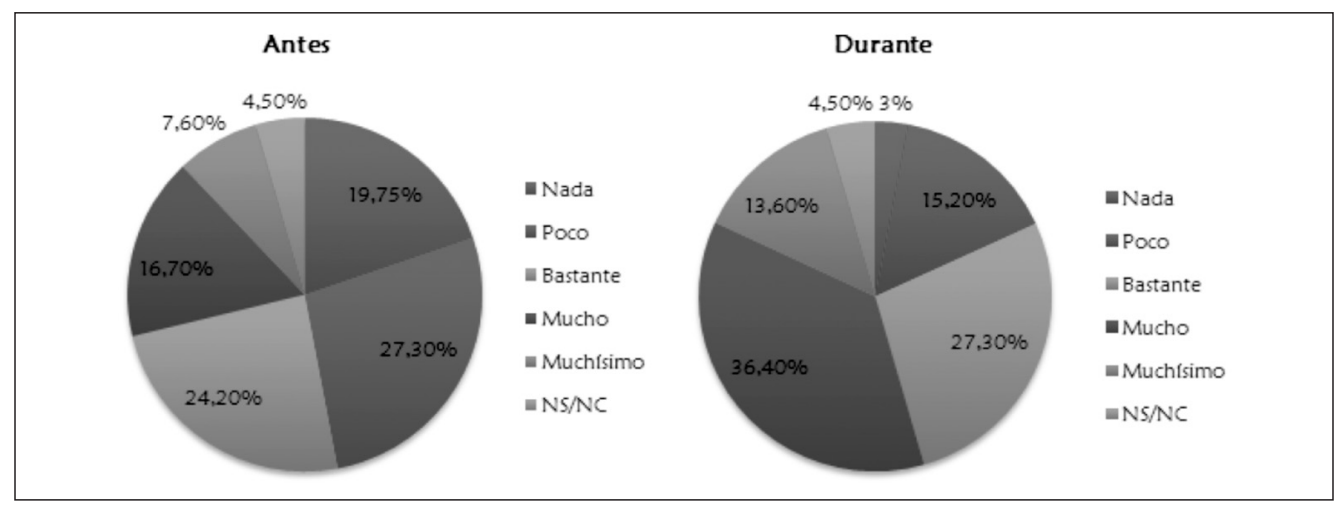

FIGURA 3.7

PORCENTAJE DE LAS RESPUESTAS DE LOS ESTUDIANTES ESPAÑOLES SOBRE LA EMPATÍA HACIA LOS MIEMBROS DE LA OTRA CULTURA

En la figura 3.7 podemos observar los porcentajes de las respuestas de los estudiantes Erasmus españoles antes y durante la estancia en el extranjero a la pregunta: « ¿Crees que puedes ponerte en el lugar de los alemanes, es decir, escucharlos y entender sus problemas y motivaciones (tener empatía)?». Durante la estancia en el extranjero, los porcentajes de «nada»y «poco» disminuyen de forma notable, mientras que «mucho», «bastante»y «muchísimo» aumentan notoriamente.

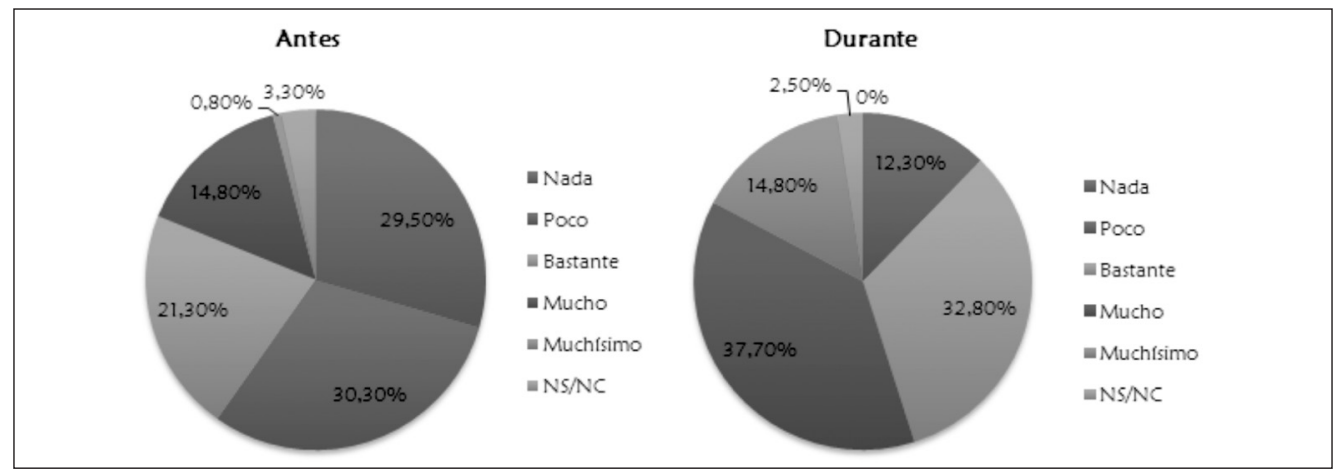

FIGURA 3.8

PORCENTAJE DE LAS RESPUESTAS DE LOS ESTUDIANTES ALEMANES SOBRE LA CAPACIDAD PARA INTEGRAR HÁBITOS DE LA CULTURA ESPAÑOLA ANTES Y DURANTE LA ESTANCIA ERASMUS 
En la figura 3.8 se presentan los porcentajes de las respuestas de los estudiantes Erasmus alemanes antes y durante la estancia en España respecto a la pregunta: « ¿Eres capaz de integrar en tu estilo de vida hábitos de la cultura española (alimentación, forma de vivir, pensar, actuar, etc.)?». De la misma forma que los estudiantes Erasmus españoles, las respuestas de los estudiantes alemanes que muestran mayores porcentajes, durante la estancia Erasmus, son «mucho», «bastante» y «muchísimo».

A continuación se presenta un resumen de las opiniones que los estudiantes españoles y alemanes aportaron en el foro de discusión, contrastada con los datos estadísticos obtenidos a partir de la encuesta.

En referencia a los estereotipos, los estudiantes Erasmus españoles afirman que, durante su estancia en Alemania, los estereotipos y prejuicios sobre la cultura alemana son menores que antes de su estancia, mientras que los estudiantes Erasmus alemanes muestran un proceso a la inversa. Estos últimos manifiestan que los estereotipos y los prejuicios sobre la cultura española son mayores que antes de la estancia en España. Los estereotipos y los prejuicios que los estudiantes alemanes tenían sobre la cultura española, antes de su estancia en España, contenían un valor positivo, mientras que los españoles tenían una imagen estereotipada más negativa de los miembros de la cultura alemana. Hay que decir que los estudiantes Erasmus llegan al país de destino con unos estereotipos consolidados sobre esa cultura, fruto del proceso de socialización. La experiencia directa con la realidad cultural ha contribuido, por una parte, a desvanecer el estereotipo negativo de los estudiantes españoles sobre los miembros de la cultura alemana y, por otro, a reforzar los estereotipos positivos de los miembros de la cultura española. Durante su estancia Erasmus los estudiantes entienden que los estereotipos y los prejuicios son fruto de las generalizaciones que hace con frecuencia la sociedad: «Son tremendamente eficaces en el trabajo. En mi trabajo, sólo hablamos mi compañera española y yo, los demás apenas se mueven de su sitio o comentan la jugada entre ellos durante la jornada completa de 8 horas. [...] Evidentemente que son bebedores: a cualquier hora del día-noche van con una cerveza en la mano. [...] En referencia a la puntualidad: yo me he encontrado con alemanes muy puntuales que toleran que llegue 10-15 minutos tarde porque «soy española», pero ellos todavía no me han fallado» (Alba).

Referente a la adopción de actitudes que prejuzgan los comportamientos, opiniones y hábitos de la otra cultura, en ambos casos se produce un fenómeno similar. Los estudiantes alemanes y españoles afirman, en su mayoría, no adoptar actitudes que prejuzgan los comportamientos, opiniones y hábitos de la otra cultura en el periodo anterior a su estancia en el extranjero. Sin embargo, esa actitud de «prejuzgamiento» disminuye ligeramente durante la estancia en ambos grupos de informantes. Además, los estudiantes españoles comentan que la estancia en el extranjero y la convivencia con los alemanes les han permitido entender mejor su forma de actuar y de pensar en determinadas situaciones $y$, en consecuencia, desarrollar una mayor empatía hacia comportamientos y actitudes de los miembros de la otra cultura: «Otra cosa a la que empiezo a adaptarme es a lo del culto al sol. La primera vez que salió el sol en Alemania me quedé flipado con la gente, que salió en masa a la calle. Poco después volvió el invierno, me volví a resfriar y entonces ya lo entendí todo» (Ebanus).

En relación a la integración de hábitos de la otra cultura en la propia, los estudiantes españoles y alemanes muestran la misma evolución. En el periodo anterior a 
su estancia en el extranjero, no existe o apenas se da una postura de adopción de las costumbres de la otra cultura tales como hábitos de alimentación, horarios, forma de pensar y de actuar, etc. En cambio, durante la estancia en el extranjero ambos grupos de informantes afirman integrar de manera significativa los hábitos de la cultura del país de destino en su forma de vida: «En algunos casos me he acostumbrado y en otros no. El comer pronto es una de ellas, la verdad es que tiene su lógica si te levantas pronto. Otra cosa es usar la bici, la uso para ir a todos los sitios. También el ir con tu paquetón de botellas al supermercado para coger el Pfand. Quitarme las zapatillas al entrar en casa. Buscar un Biergarten en cuanto hay un rayo de solecito. Desayunar los fines de semana un buen desayuno alemán [...]. Pero hay cosas a las que no me acostumbro. La cantidad de Frikadelle y de Currywurst que se comen, no lo entiendo. Otra cosa es la falta de pescado fresco. A parte, está la cerveza, esas copas de un litro de cerveza son impensables». (Manuel)

En base al análisis empírico, en general podemos asegurar que antes de la estancia en el extranjero, los estudiantes Erasmus españoles y alemanes se enfrentan a la cultura extranjera desde una perspectiva etnocéntrica y se sitúan en la fase que Bennett (1998) denomina «defensa» (nivel inicial). Aunque estos estudiantes dicen entender comportamientos y opiniones de la otra cultura (aceptación y/o cooperación) y no adoptar actitudes que prejuzguen dichas personas; muestran poca empatía hacia el hecho intercultural y tienen dificultades para integrar los hábitos de la otra cultura. Los estudiantes se protegen frente a la diferencia cultural: son conscientes de las diferencias entre culturas, pero perciben los estereotipos, en su mayoría, como un juicio cierto. Por lo que podemos decir que durante esa etapa los estudiantes españoles y alemanes experimentan la propia cultura como centro de la realidad, e incluso, pueden llegar a percibir la diferencia cultural como una «amenaza» a la propia cultura. No obstante, el hecho de que quieran realizar una estancia Erasmus ya demuestra un interés implícito por conocer otras realidades culturales.

Durante la estancia en el extranjero los estudiantes españoles y alemanes desarrollan una actitud etnorrelativa situada en la fase de «adaptación» (Bennett, 1998). Dicen ser conscientes de la influencia de la propia cultura en la percepción de la otra y la influencia de la otra cultura en la visión de la propia proceso de conocimiento de la propia cultura y de las diferencias entre la propia cultura y la otra. Manifiestan una actitud a través de la cual mantienen la propia cultura - actitudes que prejuzgan la otra cultura, etapa imprescindible para la adaptación cultural - y con la que exploran estrategias de reconocimiento de los problemas culturales o métodos de resolución de conflictos. También muestran una mayor empatía y comprensión hacia comportamientos y opiniones de las personas de la otra cultura diferentes a la suya (véase también: Ruben y Kealey, 1979; Arthur y Bennett, 1995; Müller y Gelbrich, 2004) e integran hábitos de la otra cultura (alimentación, horarios, etc.) en su forma de vida (adaptación social). En esta etapa, son capaces de elaborar un «estereotipo efectivo» (Adler, 1987) y «generalización creativa» (Mead, 1994) gracias a la interacción con los individuos de la otra cultura. Se trata, por lo tanto, de un cambio de marco de referencia cultural en el que la propia visión del mundo se amplía para incluir otras visiones, lo que Santacreu y Albert (2004) denominan «aculturación». El hecho de enfrentarse a otras realidades culturales - el creciente contacto con miembros pertenecientes a otras 
culturas, no solo con los del país de recepción - promueve la sensibilidad cultural, la apertura mental y el cambio de perspectiva cognitiva hacia una actitud etnorrelativa (véase también: Imahori y Lanigan, 1989; Arthur y Bennett, 1995; Lievens, Harris, Van Keer y Bisqueret, 2003; Mol, Born, Willemsen y Van der Molen, 2005; Shaffer, Harrison, Gregersen, Black y Ferzandi, 2006).

\section{CONCLUSIONES}

La presente investigación aporta informaciones significativas relativas al desarrollo de la competencia intercultural de los estudiantes Erasmus españoles y alemanas durante su estancia Erasmus. En relación a los objetivos planteados se puede confirmar que la estancia en el extranjero implica un proceso de adquisición complejo y multidimensional (Bolten, 2007) y, a la vez, de aplicación de conocimientos (como información relativa a esa cultura y conciencia sociolingüística), de habilidades (como la reflexión sobre las diferencias culturales y la superación de estereotipos y prejuicios) y de actitudes (como la empatía, el respeto, la aceptación de la diferencia y la adopción de costumbres de la otra cultura en la propia), que promueve el desarrollo de la competencia intercultural (Deardorff , 2009).

En base a los resultados, presentamos propuestas que tienen por objetivo, por una parte, concretar las necesidades derivadas del mismo trabajo investigador $\mathrm{y}$, por otra, sugerir recomendaciones con la finalidad de abrir camino a futuras investigaciones.

- Se requiere que el currículo y los planes de estudio de las facultades de las universidades europeas contemplen la obligatoriedad y/o posibilidad de realizar parte de su formación académica en el extranjero con una estancia mínima de cuatro meses.

- Es necesario que las instituciones también incluyan en el currículo una formación obligatoria previa a la estancia en el extranjero, cuyos objetivos y contenidos de aprendizaje se dirijan al desarrollo de la competencia intercultural y que considere «internet y sus aplicaciones (como las plataformas de aprendizaje y la Web 2.0) como herramienta base que permite crear escenarios interactivos y vías de trabajo cooperativo en forma vitural» (Bolten, 2010: 13).

- Es imprescindible facilitar y flexibilizar el reconocimiento de las cualificaciones profesionales comparables (ECTS) para incrementar la movilidad a nivel europeo.

- Es preciso valorar los recursos estructurales necesarios y definir los planes de actuación a largo plazo para poder llevar a cabo de forma adecuada el proyecto de ampliación.

A pesar de que nuestra investigación respondido los objetivos planteados somos conscientes de que el estudio tiene limitaciones por sus grandes dimensiones y que creemos que son necesarias exponer. En relación al proceso investigador, la muestra se ha limitado a estudiantes de cinco universidades españolas y de cuatro universidades alemanas, lo que supone una limitación, dado el alcance del fenómeno Erasmus. Aún así, la muestra es suficientemente representativa de la población total. Los instrumentos de recogida de datos se han basado principalmente en datos cuantitativos y cualitativos que se desprenden de los cuestionarios y de los foros 
de discusión, los cuales se podrían haber completado con otros instrumentos como puede ser la entrevista.

Para concluir, creemos conveniente que la Comisión Europea considere nuestra investigación como apoyo al desarrollo del Programa de Aprendizaje Permanente y a su difusión en las instituciones europeas de educación superior en calidad de marco estratégico común.

\section{REFERENCIAS}

Adler, P.S. (1987). Culture shock and the cross-cultural learning experience. En I. F. Luce \& E. C. Smith (Eds.), Toward internationalism (pp. 24-35). Cambridge, England.: Newbury House Publications.

Arthur, W., \& Bennett, W. (1995). The international assignee: The relative importance of factors perceived to contribute to success. Personnel Psychology, 48, 99-114.

Bennett, M. J. (1998). Intercultural communication: A current perspective. En M. J. Bennett (Coord.), Basic concepts of intercultural communication: A reader (pp. 1-34). Yarmouth, Canada: Intercultural Press.

Bennett, M.J. (1986). A developmental approach to training for intercultural sensivity. International Journal of Intercultural Relations, 10 (2), 179-196.

Bolten, J. (2010). Das Internet als basis transnationalen und interkulturellen lernens. Der «Intercultural Campus» als beispiel. Das Wort. Germanistisch Jahrbuch Russland 2010, 13-28.

Bolten, J. (2007). Was heißt «Interkulturelle Kompetenz?» Perspektiven für die internationale Personalentwicklung. En V. Künzer \& J. Berninghausen (Eds.), Wirtschaft als interkulturelle Herausforderung (pp. 21-42). Frankfurt, Deutschland: IKO.

Brislin, R. W. (1981). Cross-cultural encounters. Face-to-face encounters. New York, NY: Pergamon.

Chen, G. M., \& Starosta, W. G. (1998). Foundations of Intercultural Communication. Needham, MA: Mass.

Deardorff, D. K., Pysarchik, D., \& Yun, Z. (2009). Towards effective international learning zssessment: Principles, design and implementation. En H. de Wit (Ed.), Measuring Success in the Internationalization of Higher Education (pp. 1-8). Amsterdam, Netherlands: EAIE.

Deardorff, D. K. (2006). Policy paper zur interkulturellen kompetenz. Gütersloh, Deutschland: Bertelsmann Stiftung.

Deardorff, D.K. (2004). The identification and assessment of intercultural competencie as a student outcome of internationalization at institutions of higher education in the United States North Carolina State University (Informe de investigación). Recuperado de http://www.lib.ncsu.edu/theses/available/etd-06162004000223/unrestricted/etd.pdf

Fritz, W.; Möllenberg, A., \& Chen, G. M. (2002). Measuring Intercultural Sensitivity in different cultural contexts. Intercultural Communication Studies, 11 (2), 165-176.

Gertsen, M. C. (1990). Intercultural competence and expatriates. The International Journal of Human Resource Management, 1 (3), 341-362.

Goetz, J. P., \& LeCompte, M. D. (1988). Etnografía y diseño cualitativo en investigación educativa. Madrid, España: Morata. 
Gunzenhäuser, R., \& Haas, E. (2006). Promovieren mit plan-ihr individueller weg: von der themensuche zum doktortitel. Frankfurt, Deutschland: Verlag Barbara Budrich - UTB. Imahori, T., \& Lanigan, M. (1989). Relational model of intercultural communication competence. International Journal of Intercultural Relations, 13 (3), 269-286.

Lievens, F., Harris, M.M., Van Keer, E., \& Bisqueret, C. (2003). Predicting cross-cultural training performance: The validity of personality, cognitive ability, and dimensions measured by an assessment center and a behavior description interview. Journal of Applied Psychology, 88, 476-489.

Löschmann, M. (2001). «Was tun gegen Stereotype». En G. Wazel (Ed.), Interkulturelle Kommunikation in Wirtschaft und Fremdspracheunterricht. Frankfurt, Deutschland: Lang.

Mead, R. (1994). International management: Cross-cultural dimensions. Cambridge, England: Blackwell Publisher.

Mol, S. T., Born, M. P., \& Van der Molen, H. T. (2005). Developing criteria for expatriate effectiveness: Time to jump off the adjustment bandwagon. International Journal of Intercultural Relations, 29, 339-353.

Müller, S., \& Gelbrich, K. (2004). Interkulturelles Marketing. München, Deutschland: Verlag Vahlen.

Roy, L. (2006). In search of the global graduate: Transforming international experience into Intercultural competence (Tesis Doctoral). Recuperado de http://spectrum.library. concordia.ca/9128/1/ MR20658.pdf

Ruben, B., \& Kealey, D. (1979). Behavioral assessment of communication competency and prediction of cross-cultural adaptation. International Journal of Intercultural Relations, 3 (1), 15-27.

Ruiz, C. (1998). Instrumentos de investigación educativa. Barquisimeto, Venezuela: CIDEG.

Santacreu, O. A., \& Albert, M. C. (2004). Las dimensiones de la identidad europea [Monográfico]. Recuperado de http://www.obets.ua.es/pioneur/bajaarchivo_public. php?iden=145

Shaffer, M. A., Harrison, D. A., Gregersen, H., Black, J. S., \& Ferzandi, L. A. (2006). You can take it with you: Individual differences and expatriate effectiveness. Journal of Applied Psychology, 91 (1), 109-125.

Straffon, D. A. (2003). Assessing the intercultural sensitivity of high school students attending an international school. International Journal of Intercultural Relations, 27, 487-501.

Triandis, H. C. (1977). Interpersonal behavior. Monterrey, CA: Brooks/Cole.

Fecha de recepción: 17 de octubre de 2011.

Fecha de revisión: 19 de octubre de 2011.

Fecha de aceptación: 20 de febrero de 2012. 